\title{
LA CALIDAD PERCIBIDA DESDE LA PERSPECTIVA DE GÉNERO EN UN HOSPITAL DE COMPLEJIDAD ASISTENCIAL MÁXIMA
}

Pilar Arrizabalaga, Guillermina Orduna y Manel Santiñá

Hospital Clínic de Barcelona

parriza@clinic.ub.es

Recibido: 09-02-2010

Aceptado: 23-04-2010

\section{Resumen}

Objetivo: Analizar la calidad percibida en un hospital de tercer nivel desde la perspectiva de género. Métodos: Análisis de los cuestionarios de satisfacción a los/las pacientes ingresados/as durante octubre y noviembre 2003-2005 con el test de la T de Student-Fisher y la prueba de $\chi^{2}$. Resultados: De los 2660 cuestionarios, 44,7\% fueron de hombres, 55,55 \pm 17,3 años, y 53,3\% de mujeres, $48 \pm 18,4$ años. El $36 \%$ tenía estudios primarios, $18 \%$ formación profesional y el $22 \%$ universitarios. Los hombres conocían las normas de hospitalización y el nombre del médico/a mejor que las mujeres $(\mathrm{p}<0.0001)$. Las mujeres se consideraron mejor informadas sobre su enfermedad que los hombres $(\mathrm{p}<0.05)$. Ambos grupos volverían a elegir el mismo centro. Conclusión: Las diferencias en la calidad percibida entre ambos sexos sugieren que el género debería contemplarse en sistemas organizativos centrados en los cuidados del paciente.

Palabras claves: Opinión del usuario, calidad percibida, diferencias entre sexos.

\begin{abstract}
Objective: To analyse the perceived assistance quality of third level hospital inpatients, carrying out an essay that takes in account only the gender of the surveyed. Methods:
\end{abstract}


Descriptive analysis of surveys of the inpatients between October and November 2003 2005 with the $\mathrm{T}$ of Student-Fisher and the $\chi^{2}$ tests respectively. Results: From 2660 surveyed, 44,7\% were men aged 55,55 $\pm 17,3$, and 53,3\% were women aged $48 \pm 18,4$. $36 \%$ had a primary school degree, $18 \%$ professional and $22 \%$ university studies. Men were considered better informed than women about hospitalization rules and knew better the name of their doctors $(\mathrm{p}<0.0001)$. Women were better informed about their illness $(\mathrm{p}<0.05)$. There was no difference in the choice of the same centre for the future. Conclusions: The perceived assistance quality is influenced by gender, so it should be taken into account in organizing systems oriented to satisfying the patient needs.

Key words: Opinion of the user, hospital perceived quality, gender differences.

\section{Introducción}

En el marco actual de la medicina de gran complejidad tecnológica y ejercicio colectivo multidisciplinar en grandes instituciones, el proceso asistencial se determina por el conjunto de cuidados y la comunicación centrados en el/la paciente. La calidad asistencial hospitalaria se define por la satisfacción de las necesidades y de las aspiraciones del/de la paciente mediante un consumo eficiente de los recursos. Entre la calidad asistencial se distinguen dos grandes componentes: la calidad técnica y la calidad percibida. La calidad técnica incluye la evaluación de los resultados derivados del diagnóstico y del tratamiento. La calidad percibida incluye la evaluación de la satisfacción y las expectativas del/de la paciente y de sus familiares.

Por tanto, la opinión de los/as pacientes dirigida a conocer sus necesidades y sus expectativas se presenta indispensable en los servicios sanitarios en la búsqueda de la excelencia (Ibern, 1992 y Bohigas, 1995). En este sentido, el Hospital Clínico de Barcelona viene realizando una encuesta anual (Lledó, 1998), a fin de conocer la 
opinión de los/as pacientes ingresados/as sobre los diferentes elementos que configuran la calidad percibida.

El papel de la condición sexual de los usuarios en la evaluación de la calidad percibida de los servicios sanitarios se ha reportado en el ámbito de la asistencia primaria (Saarento et al., 2000; Millar, 2001). En el ámbito hospitalario, se ha indicado que la calidad percibida también difiere entre los pacientes ingresados de uno y otro sexo en los hospitales de segundo nivel que abordan el diagnóstico y tratamiento de la patología de complejidad media el nivel correspondiente a los hospitales comarcales (Kressin et al., 1999; Ladwig et al., 2000). Sin embargo, se desconoce si el sexo del paciente pudiera jugar un papel en la calidad percibida en hospitales universitarios de tercer nivel con complejidad máxima, los que abarcan patologías y procedimientos terapéuticos muy seleccionados. En estos últimos, pudiera pensarse que la calidad técnica primara por encima de la calidad percibida $\mathrm{y}$, por tanto, sobre supuestos condicionantes del género en el proceso asistencial.

\section{Métodos}

Se trata de un estudio epidemiológico, descriptivo y transversal, a partir de los resultados de la encuesta de opinión realizada a los/as pacientes ingresados/as en el Hospital Clínic de Barcelona, un hospital universitario de tercer nivel, que incluye patología de complejidad máxima, con 774 camas. Se han analizado los resultados de 2660 cuestionarios obtenidos en el otoño de los años 2003, 2004 y 2005.

Durante los dos meses de octubre y noviembre, se realiza la encuesta de satisfacción a los/as pacientes ingresados/as tres o más días. Se exceptúan los/as pacientes ingresados/as en los servicios de psiquiatría, neonatología y unidades de cuidados críticos, para quienes se confeccionan cuestionarios específicos.

El cuestionario anónimo y voluntario se entrega al/la paciente hospitalizado/a, solicitándole que una vez completado, lo deposite en una urna dispuesta en el servicio 
de admisiones para tal fin, en el momento del alta hospitalaria (Anexo 1). El procedimiento se ha venido utilizando desde 1992, cuando se publicó el fundamento metodológico de validación de la encuesta (Lledó et al., 1992). El índice de respuesta fue del $33 \%$.

Con los resultados obtenidos se creó una base de datos que se analizó mediante el paquete estadístico SPSS versión 10.0. Se utilizaron el test de la T de Student-Fisher para las variables cuantitativas, y la prueba de $\chi^{2}$ para las variables cualitativas. El nivel de confianza y el intervalo de confianza de la muestra fueron $95 \%$ y 3,41 respectivamente.

\section{Resultados}

De la muestra analizada de 2660 pacientes, 44,7\% fueron hombres con una edad promedio de 55,55 \pm 17,3 (media \pm desviación estándar) años, y el 53,3\% fueron mujeres de $48 \pm 18,4$ años. El 68\% de los/las respondedores/as residía en Barcelona y hasta el $90 \%$ en su provincia. El nivel de estudios más frecuente en el conjunto era el nivel de primaria -36\%-, $18 \%$ había cursado formación profesional y el $22 \%$ tenía estudios universitarios. Del servicio de urgencias procedía el 58\% de los/as pacientes ingresados/as.

La Tabla 1 (anexa) recoge el resultado del análisis de la prueba del $\chi^{2}$ para las variables cualitativas. Se destaca que el porcentaje de reingresos fue significativamente superior para los hombres que para las pacientes mujeres $(\mathrm{p}<0.0001)$. Los hombres se consideraron mejor informados que las mujeres sobre las normas de hospitalización ( $\mathrm{p}<$ 0.0001), y conocían más el nombre del médico/a ( $<$ < 0.0001) y de la enfermera/o de cualquiera de los turnos (mañana, tarde y noche). Las pacientes mujeres se consideraron mejor informadas que los pacientes hombres acerca de su enfermedad ( $p<0.05)$, así como también de las pruebas complementarias diagnósticas. Las respuestas sobre los 
aspectos relativos al confort de la habitación, la limpieza y la hostelería no mostraron diferencias significativas entre ambos sexos. Sí hubo diferencias en la percepción de molestias derivadas del ruido. Los hombres las manifestaron más frecuentemente que las mujeres $(\mathrm{p}<0.05)$, pero cabe señalar que los primeros se alojaron en habitaciones individuales en menor porcentaje que las segundas.

La Tabla 2 muestra el análisis comparativo de las variables cuantitativas, edad y puntuación asignada al hospital. Los respondedores de la encuesta fueron de más edad que las respondedoras. Los hombres dieron una puntuación globalmente de los servicios sanitarios recibidos durante el ingreso hospitalario más alta que la puntuación que dieron las mujeres, pero ellos y ellas volverían a elegir el mismo centro en un futuro ingreso.

Tabla 2 - Edad y puntuación global del servicio sanitario recibido durante la hospitalización. Análisis bivariante. Test de la t de Student-Fisher

\begin{tabular}{|l|c|c|c|c|}
\hline & Escala & Varones & Mujeres & t \\
\hline 2 & Edad & $55.55(17.3)$ & $48(18.4)$ & $10.62 * * *$ \\
\hline 25 & Puntuación & $8.59(1.17)$ & $8.56(1.20)$ & $0.608 *$ \\
\hline
\end{tabular}

Los números de la primera columna corresponden al número de la pregunta del cuestionario. Los resultados se dan como media y desviación estándar entre paréntesis. $* p<0.05 ; * * p<0.001$; $* * * p<0.0001$

\section{Discusión}

Este estudio confirma diferencias en la calidad percibida en función del género del paciente ingresado, incluso en el ámbito hospitalario de máxima complejidad asistencial. Los pacientes hombres se consideraron mejor informados que las pacientes mujeres sobre las normas de hospitalización, y conocían más el nombre del médico/a y de la enfermera/o de cualquiera de los turnos de mañana, tarde y noche. Las pacientes 
mujeres conocían más que los pacientes hombres acerca de su patología, pruebas y procedimientos diagnósticos a los que debían someterse.

Al comparar las respuestas de pacientes del mismo sexo durante el período de este estudio, 2003-2005, con la misma encuesta realizada durante el periodo anterior, 1998-2000 (Santiñá et al., 2002), se aprecian diferencias relativas en cuanto al número de reingresos, la percepción de la información, la relación con los profesionales sanitarios y la percepción del confort. En el presente estudio y a diferencia del período anterior, el conjunto de las respuestas satisfactorias de las pacientes mujeres se han doblado, lo que apoya la calidad percibida por las mujeres como más positiva frente a la calidad percibida por hombres respecto a la atención hospitalaria (Mira, 2000).

Otra diferencia objetiva se ha modificado en el intervalo entre 1998-2000 y 2003-2005. El aumento de los reingresos para los pacientes hombres ha superado el 3\% mientras que para las pacientes mujeres ha disminuido el número de reingresos un $4 \%$. Varias explicaciones pudieran contribuir al aumento de los reingresos hospitalarios masculinos y al descenso de los reingresos hospitalarios femeninos. El hecho de que las mujeres sean 7 años de media más jóvenes que los hombres pudiera ser un factor, pero la inversión de los reingresos hospitalarios entre ambos sexos pudiera reflejar el mejor conocimiento y manejo de la enfermedad que expresan las mujeres como se desprende del presente estudio. Además, factores sociales y culturales, que han llevado a las mujeres a una mayor sensibilidad y apreciación de su salud con la consiguiente mejoría de los estilos de vida y de las prácticas preventivas, como son las relativas al consumo de tóxicos - tabaco y alcohol - que hoy por hoy aún es menor en las mujeres que en los hombres (Borrell et al., 2006).

La percepción del silencio manifestada por los pacientes hombres ha disminuido llamativamente entre ambos períodos, $13 \%$. En cambio, el descenso apenas ha llegado al $3 \%$ entre las pacientes mujeres. La diferencia apoyaría la mayor exigencia de la condición femenina en aspectos tangibles de la asistencia hospitalaria (GonzálezValentín, Padín y Ramón, 2005; Guix, Fernández y Sala, 2006), en el mismo sentido 
que sucede en el ámbito de la asistencia primaria (Street et al., 2005; Weisman et al., 2000).

Algunos autores han indicado que la juventud atenúa las diferencias de la calidad percibida entre ambos sexos, en particular entre los pacientes menores de 25 años, en los que otros factores como el dolor o la ansiedad tienen mayor peso en la satisfacción de los servicios sanitarios (Rahmqvist, 2001). Sin embargo, el $60 \%$ de la asistencia hospitalaria corresponde a patología crónica que alcanza a más de un tercio de la población mayor de 65 años (El Médico Interactivo, 2008), y, que requiere de dos o más reingresos hospitalarios en más del $50 \%$ de los pacientes (González et al., 2008). En consecuencia, las diferencias entre ambos sexos en los distintos aspectos que configuran la calidad asistencial percibida adquieren relevancia para la excelencia en la atención hospitalaria. Incluso en los servicios sanitarios de máxima complejidad, las características bio-psico-sociales - el género - del paciente deberían contemplarse a fin de la excelencia orientada a los cuidados centrados en el paciente.

\section{Conflicto de intereses:}

Los autores declaran que no hay conflicto de intereses en la realización del estudio ni en la elaboración del manuscrito. 


\section{BIBLIOGRAFÍA}

- Bohigas, L. (1995): “La satisfacción del paciente”. En Gaceta Sanitaria, n 50, pp. 284-286.

- Borrell, C.; Benach, J.; Grupo de trabajo CAPS-FJ Bofill. (2006): “La evolución de las desigualdades en salud en Cataluña”. En Gaceta Sanitaria, no 20, pp. 396-406.

- El Médico Interactivo. Diario Electrónico de la Sanidad. (2008): “I Congreso Nacional de Atención Sanitaria al Paciente Crónico”. En El Médico Interactivo, nº 2111, 13/15 de septiembre, [en línea] Disponible en: http://www.medynet.com [18/02/2010].

- González, N. et al. (2008): "Satisfacción de los usuarios de cuatro hospitales del Servicio Vasco de Salud”. En Gaceta Sanitaria, no 22, pp. 210-217.

- González-Valentín, A.; Padín-López, S. y Ramón-Garrido, E. (2005): "Patient satisfaction with nursing care in a regional university hospital in Southern Spain". En Journal of Nursing Care Quality, nº 20, pp. 63-72.

- Guix Oliver, J.; Fernández Ballart, J. y Sala Barbany, J. (2006): “Patients, physicians and nurses: three different points of view on the same issue. Attitudes to and perceptions of patient rights". En Gaceta Sanitaria, n 6, pp. 465-472.

- Ibern Regás, P. (1992): “La mesura de la satisfacció en els serveis sanitaris”. En Gaceta Sanitaria, nº 6, pp. 176-185.

- Kressin, N. R. et al. (1999): "Patient satisfaction with Department of Veterans Affairs health care: do women differ from men?”. En Military Medicine, n 164, pp. 283-288.

- Ladwig, K. H. et al. (2000): "Gender differences in emocional disability and negative health perception in cardiac patients 6 months after stent implantation". En Journal of Psychosomatic Research, $\mathrm{n}^{\mathrm{o}}$ 48, pp. 501-508.

- Lledó, R. y Prat, A. (1998): “Calidad Asistencial”. En: M. A. Asenjo (ed): Gestión diaria del hospital. Barcelona: Masson, pp. 285-305.

- Lledó, R. et al. (1992): "La importancia de las encuestas de opinión a los usuarios del hospital sobre la calidad asistencial”. En Todo Hospital, no 87, pp. 9-11. 
- Millar, M. (2001): "Patient satisfaction with general practice in Ireland". En Irish Medical Journal, $\mathrm{n}^{\circ}$ 94, pp. 108-109.

- Mira, J. J. y Aranaz, J. (2000): “La satisfacción de paciente como una medida del resultado de la atención sanitaria”. En Medicina Clinica, nº 114 Supl 3, pp. 26-33.

- Rahmqvist, M. (2001): "Patient satisfaction in relation to age, health status and other background factors: a model for comparisons of care units". En International Journal for Quality in Health Care, nº 13, pp. 385-390.

- Saarento, O. et al. (2000): "Sex differeces in the contact rates and utilization of psychiatric services. A three-year follow-up study in Northern Finland”. En European Psychiatry, $\mathrm{n}^{\mathrm{o}}$ 15, pp. 205-212.

- Santiñá, M. et al. (2002): “Calidad percibida y sexo de los pacientes atendidos en un hospital universitario”. En Revista de Calidad Asistencial, no 17, pp. 218-223.

- Street, R. L. et al. (2005): "Patient participation in medical consultations: why some patients are more involved than others". En Medical Care, n 43, pp. 960-969.

- Weisman, C. S. et al. (2000): “Gender and patient satisfaction with primary care: tuning in to women en quality measurement". En Journal of Women's Health \& Gender-Based Medicine, $\mathrm{n}^{\circ}$ 9, pp. 657-665. 


\section{ANEXO I \\ Cuestionario de opinión al usuario}

1- Persona que contesta la encuesta:

Propio enfermo $\square \quad$ Cónyuge - pareja $\square \quad$ Padre - Madre $\square \quad$ Hijo-Hija $\square$ Otros $\square$

2- Edad de la persona que contesta la encuesta......... años.

3- Sexo de la persona que contesta la encuesta:

Hombre $\square \quad$ Mujer $\square$

4- País de origen de la persona que responde la encuesta.........

5- Lugar de residencia de la persona que contesta la encuesta

Barcelona Ciudad $\square$ Resto de la Provincia $\square$ Resto Cataluña $\square$ Otros $\square$

6- Nivel de estudios de la persona que contesta la encuesta

Ninguno $\square \quad$ Primarios (EGB, ESO) $\square \quad$ Formación profesional $\square$

Bachillerato superior $\square \quad$ Universitarios $\square$

7- ¿Había estado ingresado/a anteriormente en este hospital?

$\mathrm{Si} \square$

No $\square$

8- ¿Cómo ingresó en el Hospital Clínico?

Después de una visita a Urgencias $\square$

Tenía el ingreso programado $\square$

9- ¿En qué Servicio / Especialidad ha estado ingresado/a?

10- La duración de su estancia en el hospital, la considera:

Insuficiente $\square \quad$ Suficiente/Correcta $\square \quad$ Excesiva $\square$

11- Tanto si ha ingresado por Urgencias o con una programación previa, cómo valora los siguientes aspectos en el proceso de la admisión hospitalaria:

\begin{tabular}{|l|l|l|l|l|l|}
\hline & Muy buena & Buena & Regular & Mala & Muy Mala \\
\hline Trato recibido & & & & & \\
\hline Información recibida & & & & & \\
\hline Agilidad de los trámites & & & & & \\
\hline
\end{tabular}

12- Usted ha sido ingresado/a en una habitación:
Individual $\square$
Doble $\square$
Otros $\square$

13- Al llegar a la habitación, ¿le explicaron las normas y el funcionamiento de la planta de hospitalización? Si $\square \quad$ No $\square$

14- ¿cómo valora el trato que ha recibido por parte del personal?

\begin{tabular}{|l|l|l|l|l|l|}
\hline & Muy Bueno & Bueno & Regular & Malo & Muy Malo \\
\hline Médico & & & & & \\
\hline Enfermería & & & & & \\
\hline Camilleros & & & & & \\
\hline Limpieza & & & & & \\
\hline Limpieza & & & & & \\
\hline
\end{tabular}

15- Cómo considera la información que ha recibido a los siguientes aspectos:

\begin{tabular}{|l|c|c|c|c|c|}
\hline & Muy Bueno & Bueno & Regular & Malo & Muy Malo \\
\hline Característica de & & & & & \\
\hline
\end{tabular}




\begin{tabular}{|l|l|l|l|l|l|}
\hline la enfermedad & & & & & \\
\hline $\begin{array}{l}\text { Pruebas } \\
\text { complementarias } \\
\text { realizadas }\end{array}$ & & & & & \\
\hline $\begin{array}{l}\text { Tratamientos y } \\
\text { controles a seguir }\end{array}$ & & & & & \\
\hline
\end{tabular}

16- Su valoración de las instalaciones y servicios generales del centro es:

\begin{tabular}{|l|l|l|l|l|l|}
\hline & Muy Bueno & Bueno & Regular & Malo & Muy Malo \\
\hline $\begin{array}{l}\text { Silencio en } \\
\text { dependencias del } \\
\text { Hospital }\end{array}$ & & & & & \\
\hline Limpieza & & & & & \\
\hline Lavandería & & & & & \\
\hline $\begin{array}{l}\text { Confort de la } \\
\text { habitación }\end{array}$ & & & & & \\
\hline El horario & & & & & \\
\hline
\end{tabular}

17- En relación con la comida proporcionada, cómo calificaría los siguientes aspectos:

\begin{tabular}{|l|l|l|l|l|l|}
\hline & Muy Buena & Buena & Regular & Mala & Muy Mala \\
\hline Presentación & & & & & \\
\hline Temperatura & & & & & \\
\hline Gusto & & & & & \\
\hline Calidad & & & & & \\
\hline Horario & & & & & \\
\hline
\end{tabular}

18- ¿Conoce el nombre del médico/a responsable de su asistencia?

Si $\square \quad$ No $\square$

19- ¿Conoce el nombre del enfermero/a de turno?

$\begin{array}{lllll}\text { Mañana } & & \text { Tarde } & \text { Noche } \\ \text { Si } \square & \text { No } \square & \text { Si } \square & \text { No } \square & \text { Si } \square\end{array}$

20- ¿Los procedimientos informativos durante su estancia han sido suficientes?

Si $\square \quad$ No $\square$

22- ¿Considera que ha estado garantizada su confidencialidad de la información referente a su persona?

Si $\square \quad$ No $\square$

23- ¿Considera que ha sido tratado/a con respecto hacia su dignidad humana y su intimidad personal?

Si $\square \quad$ No $\square$

24- En caso necesario: ¿escogería nuestro Hospital para ingresar otra vez?

Si $\square \quad$ No $\square$

25- ¿Cómo puntuaría globalmente el servicio sanitario que ha recibido durante su hospitalización?

\begin{tabular}{|c|c|c|c|c|c|c|c|c|c|}
\hline \multicolumn{2}{|c|}{ Muy Satisfactoria } & \multicolumn{2}{|c|}{ Satisfactoria } & \multicolumn{2}{c|}{ Indiferente } & \multicolumn{2}{c|}{ Insatisfactoria } & \multicolumn{2}{c|}{ Muy Insatisfactoria } \\
\hline 10 & 9 & 8 & 7 & 6 & 5 & 4 & 3 & 2 & 1 \\
\hline
\end{tabular}

26- Sugerencias y comentarios: 
Tabla 1 - Características sociodemográficas y calidad percibida de los/as pacientes. Análisis bivariante. Test de $\mathrm{chi}^{2}$

\begin{tabular}{|c|c|c|c|c|}
\hline & Pregunta & Varones $(n=1213)$ & Mujeres $(n=1447)$ & $\chi^{2}$ \\
\hline \multirow[t]{3}{*}{7} & Reingreso & & & \\
\hline & $\mathrm{Si}$ & 60.2 & 48,7 & \\
\hline & No & 13 & 51,3 & \\
\hline \multirow[t]{3}{*}{8} & Vía ingreso & & & \\
\hline & Urgencias & 58.8 & 58.6 & 0.017 \\
\hline & Programado & 41.2 & 41.4 & \\
\hline \multirow[t]{4}{*}{10} & Duración & & & \\
\hline & Insuficiente & 5.5 & 6.8 & $13.69 * *$ \\
\hline & Suficiente & 90.1 & 91.2 & \\
\hline & Excesivo & 4.4 & 2 & \\
\hline \multirow[t]{12}{*}{11} & Trato de Admisión & & & \\
\hline & Bien/Muy Bien & 97 & 97.2 & \\
\hline & Regular & 2.4 & 2.5 & \\
\hline & Mal/Muy Mal & 0.2 & 0.2 & \\
\hline & Información de Admi & & & \\
\hline & Bien/Muy Bien & 86.1 & 87.7 & \\
\hline & Regular & 11.1 & 10.4 & \\
\hline & Mal/Muy Mal & 2.8 & 1.8 & \\
\hline & Agilidad en Trámites & & & \\
\hline & Bien/Muy Bien & 83.6 & 85.8 & \\
\hline & Regular & 14 & 11 & \\
\hline & Mal/Muy Mal & 2.6 & 3.2 & \\
\hline \multirow[t]{3}{*}{12} & Habitación & & & \\
\hline & Individual & 11 & 17.4 & $40.63^{* * *}$ \\
\hline & Doble & 80.4 & 78.6 & \\
\hline \multirow[t]{3}{*}{13} & Explicar & & & \\
\hline & $\mathrm{Si}$ & 73 & 64.6 & \\
\hline & No & 27 & 35.4 & \\
\hline \multirow[t]{15}{*}{14} & Trato médico & & & \\
\hline & Bueno/Muy Bueno & 97 & 96.6 & 2.03 \\
\hline & Regular & 2.7 & 3 & \\
\hline & Mal/Muy Mal & 0.5 & 0.4 & \\
\hline & Trato Enfermería & & & \\
\hline & Bueno/Muy Bueno & 97.7 & 98 & 1.03 \\
\hline & Regular & 2 & 1.7 & \\
\hline & Malo/Muy Malo & 0.4 & 0.2 & \\
\hline & Trato Camillero & & & \\
\hline & Bueno/Muy Bueno & 95 & 97.7 & $17.26^{* * *}$ \\
\hline & Regular & 4 & 2.1 & \\
\hline & Malo/Muy Malo & 1 & 0.2 & \\
\hline & Trato Limpieza & & & \\
\hline & Regular & 5.5 & 6 & \\
\hline & Malo/Muy Malo & 0.7 & 1 & \\
\hline \multirow[t]{3}{*}{15} & Entender explicacion & nedad & & \\
\hline & Bueno/Muy Bueno & 87.5 & 90.4 & $9.74 *$ \\
\hline & Regular & 9.5 & 7.5 & \\
\hline
\end{tabular}




\begin{tabular}{|c|c|c|c|c|}
\hline & Malo/Muy Malo & 3 & 2.1 & \\
\hline \multicolumn{5}{|c|}{ Pruebas Complementarias } \\
\hline & Bueno/Muy Bueno & 90.4 & 92.7 & 5.58 \\
\hline & Regular & 7.3 & 6 & \\
\hline & Malo/Muy Malo & 2.4 & 1.4 & \\
\hline \multicolumn{5}{|c|}{ Tratamiento y controles } \\
\hline & Bueno/Muy Bueno & 91 & 93.2 & 7.96 \\
\hline & Regular & 7.2 & 5.4 & \\
\hline & Malo/Muy Malo & 1.7 & 1.4 & \\
\hline 16 & \multicolumn{4}{|l|}{ Silencio } \\
\hline & Bueno/Muy Bueno & 67 & 73 & $12.29 *$ \\
\hline & Regular & 26 & 21.7 & \\
\hline & Malo/Muy Malo & 7 & 5.2 & \\
\hline \multicolumn{5}{|c|}{ Limpieza } \\
\hline & Bueno/Muy Bueno & 91 & 91 & 1.13 \\
\hline & Regular & 8.3 & 8 & \\
\hline & Malo/Muy Malo & 0.7 & 1 & \\
\hline \multicolumn{5}{|c|}{ Confort Habitación } \\
\hline & Bueno/Muy Bueno & 76 & 78.4 & 2.72 \\
\hline & Regular & 20 & 17.7 & \\
\hline & Malo/Muy Malo & 4 & 4 & \\
\hline \multicolumn{5}{|c|}{ Visitas } \\
\hline & Bueno/Muy Bueno & 93.3 & 95 & 3.70 \\
\hline & Regular & 5.4 & 4 & \\
\hline & Malo/Muy Malo & 1.4 & 1 & \\
\hline \multirow[t]{4}{*}{17} & Comida & & & \\
\hline & Bueno/Muy Bueno & 71.4 & 71 & 1.22 \\
\hline & Regular & 22.4 & 22 & \\
\hline & Malo/Muy Malo & 6 & 6.3 & \\
\hline \multirow[t]{3}{*}{18} & Nombre Médico/a & & & \\
\hline & $\mathrm{Si}$ & 78 & 68 & \\
\hline & No & 22 & 32 & \\
\hline \multirow[t]{3}{*}{19} & Nombre Enfermero/a & & & \\
\hline & $\mathrm{Si}$ & 71 & 67 & \\
\hline & No & 29 & 33 & \\
\hline \multirow[t]{3}{*}{20} & Procedimientos informa & & & \\
\hline & $\mathrm{Si}$ & 89.5 & 89 & \\
\hline & No & 10.5 & 11 & \\
\hline \multirow[t]{3}{*}{21} & Explicar & & & \\
\hline & $\mathrm{Si}$ & 95 & 94 & \\
\hline & No & 5 & 6 & \\
\hline \multirow[t]{3}{*}{23} & Dignidad/Intimidad & & & \\
\hline & $\mathrm{Si}$ & 97 & 96 & \\
\hline & No & 3 & 4 & \\
\hline \multirow[t]{3}{*}{24} & Escogería nuevamente & & & \\
\hline & $\mathrm{Si}$ & 98 & 98 & \\
\hline & No & 2 & 2 & \\
\hline
\end{tabular}

Los números de la primera columna corresponden al número de la pregunta del cuestionario. Los resultados se dan en porcentaje. * $p<0.05 ; * * p<0.001 ; * * * p<0.0001$ 